\title{
DEMANDA ENERGÉTICA DE UM SUBSOLADOR ADAPTADO PARA DEPOSIÇÃO DE MATERIAL POROSO EM DRENOS LIVRES
}

Haroldo C. Fernandes ${ }^{1}$, Roberto C. Orlando², Daniel M. Leite ${ }^{3}$, Marcelo M. Costa ${ }^{4}$, Marconi Ribeiro Furtado Júnior ${ }^{5}$

\section{RESUMO}

O objetivo deste trabalho foi avaliar a demanda energética de um protótipo de um subsolador com torpedo para inserção de material poroso em drenos. Os testes de avaliação do desempenho do protótipo foram efetuados em uma área experimental da Universidade Federal de Viçosa, localizadas no município de Viçosa-MG. O material poroso utilizado foi a casca de arroz. Foi mensurada a potência requerida e consumo de combustível. O delineamento experimental utilizados foi o fatorial, que consistiu em 2 velocidades e 4 profundidades com 3 repetições. O solo apresentou um perfil argiloso, caracterizado como Podzólico Vermelho-Amarelo. O maior requerimento de força pelo implemento foi de $13711,1 \mathrm{~N}$ e a maior potência de $17,85 \mathrm{~kW}$. Esses valores foram observados para a velocidade de 4,9 $\mathrm{km} \mathrm{h}^{-1}, \mathrm{na}$ profundidade de $0,60 \mathrm{~m}$. A força média requerida pelo protótipo foi menor do que a encontrada na literatura. A média da potência requerida pelo implemento foi $9,98 \mathrm{~kW}$, e pelo conjunto trator-implemento de 12,26 kW. A relação força de tração/profundidade de trabalho apresentou-se dentro do limite estabelecido pela ASAE para solos argilosos.

Palavras-chave: drenagem, potência, subsolagem.

\section{ABSTRACT \\ ENERGY DEMAND OF A SUBSOILER ADAPTED FOR DEPOSITION OF POROUS MATERIAL IN FREE DRAINS}

This study was done to evaluate energy demand of a prototype subsoiler with expander to insert porous materials into drains. The study was done in an experimental area of the Federal University of Viçosa, Viçosa-MG. Fuel consumption and power requirement were measured using rice hull as the test porous material. The experiment was done with three replications in the factorial design, consisting of two speeds and four depths. The clayey soil profile was characterized as red-yellow podzol. The maximum force demanded by the prototype was $13711.1 \mathrm{~N}$ with maximum power of $17.85 \mathrm{~kW}$. at the speed of $4.9 \mathrm{~km} \cdot \mathrm{h}^{-1}$ and the depth of $0.60 \mathrm{~m}$. The mean force required by this prototype was lower than that reported in the literature. The average power required by the subsoiler was $9.98 \mathrm{~kW}$, and that of the tractor-prototype combine was $12.26 \mathrm{~kW}$. The tensile strength / work depth ratio was within the limits established by ASAE for clay soils.

Keywords: drainage, power, subsoiling.

\section{Recebido para publicação em 11/11/2010. Aprovado em 26/03/2012.}

1- Eng. Agrícola, Professor Associado, DEA - UFV, Viçosa-MG, haroldo@ufv.br

2- Eng. Agrícola, Professor Adjunto, Faculdade de Ciências Agrárias - UFGD, Dourados-MS, rc_orlando@gmail.com

3- Lic. em Ciências Agrícolas, Doutorando em Engenharia Agrícola, DEA - UFV, Viçosa-MG, daniel.mariano@ufv.br

4- Eng. Agrônomo, Doutorando em Engenharia Agrícola, DEA- UFV, Viçosa-MG, marcelo.costa@ufv.br

5- Eng. Agrônomo, Mestrando em Engenharia Agrícola, DEA - UFV, Viçosa-MG, marconi.junior@ufv.br 


\section{INTRODUÇ̃̃O}

A subsolagem tem por objetivo desestruturar camadas compactadas do solo. Isso proporciona um melhor desenvolvimento radicular e aumento da produção agrícola (GROTTA et al., 2004). Os subsoladores também podem ser utilizados em solos encharcados, com o intuito de estabelecer canais para a drenagem do excesso de água. Esse tipo de subsolador apresenta uma estrutura chamada torpedo, que permite a formação de um canal para escoamento da água, na profundidade de atuação do implemento. O preenchimento deste canal com material poroso, como casca de arroz, permite a funcionalidade desses drenos por um maior período de tempo.

Um subsolador convencional pode ser adaptado para introduzir material poroso no solo para formação de drenos. Pode-se aproveitar restos culturais que apresentam alta relação carbono/nitrogênio como a casca de arroz, casca de café e outros, para o estabelecimento de drenos de baixo custo utilizando subsoladores adaptados.

Em uma propriedade agrícola é importante conhecer a demanda de potência de cada implemento utilizado. Isso é necessário para determinar a potência requerida nos tratores que irão realizar as operações. Porém, a demanda de potência em operações agrícolas depende de vários fatores, tais como características do implemento, tipo de solo, teor de água, conteúdo de argila, profundidade de trabalho, velocidade de trabalho, entre outros (SALVADOR et al., 2008).

Segundo Plasse et al. (1985), dentre as propriedades físicas do solo, o teor de água é a que tem maior influência sobre a operação de subsolagem. Martucci (1985) também confirma que o teor de água no solo influencia consideravelmente o consumo de energia na subsolagem. Para este autor, solos com baixo teor de água representam as condições ideais para esta operação.

Summers et al. (1986) estudaram a força de tração em função da velocidade de deslocamento para alguns equipamentos de preparo de solo. Os autores observaram que a força de tração é diretamente proporcional à velocidade, para os arados de cinzel (subsoladores com profundidade de trabalho de até $400 \mathrm{~mm}$ ), grades de disco e escarificadores. Para os arados de aivecas, a força de tração variou com a velocidade segundo uma função quadrática. Além disso, observa-se que a profundidade de trabalho influencia linearmente na força requerida na barra de tração.

Em relação ao teor de argila do solo, Santos Filho (1993) observou um crescente aumento da força de tração média necessária à subsolagem, com o aumento do teor de argila no solo.

Para Bridges e Smith (1979), máquinas maiores não representam maior consumo de energia por área. Por isso, reduzir o tamanho de implementos para melhorar a economia de combustível pode ser um erro. Isso, porque o importante é o consumo de energia requerido por área, e muitas vezes um implemento menor consome mais energia.

Hunt (1986) afirma que a área coberta por unidade de tempo é uma forma de identificar a capacidade operacional de um implemento. Entretanto, o consumo de energia serve para caracterizar economicamente uma operação de preparo, bem como a fonte de potência (trator). Assim, é possível expressar a quantidade total de energia consumida por um conjunto máquina-implemento em várias operações necessárias na atividade agropecuária. $\mathrm{O}$ cálculo da energia consumida nas operações de campo deve ser feito a partir da capacidade operacional dos implementos e máquinas, inclusive do trator. $\mathrm{O}$ autor afirma ainda que as operações de preparo do solo consomem mais da metade da potência requerida nas propriedades agrícolas.

Sendo assim, objetivou-se com este trabalho determinar a potência demandada e o consumo especifico de combustível de subsolador adaptado para deposição de material poroso em drenos livres.

\section{MATERIAL E MÉTODOS}

O protótipo do subsolador com torpedo e mecanismo para inserção de material poroso em drenos livres foi desenvolvido no Laboratório de Mecanização Agrícola (LMA). Esse laboratório pertencente ao Departamento de Engenharia Agrícola (DEA) da Universidade Federal de Viçosa, MG. Os trabalhos de avaliação foram realizados em uma área experimental da instituição, localizada nas coordenadas $42^{\circ} 52^{\prime} 40^{\prime \prime} \mathrm{W}, 20^{\circ} 45^{\prime} 20^{\prime \prime} \mathrm{S}$, a $658 \mathrm{~m}$ de altitude.

O subsolador utilizado foi desenvolvido por Orlando (1999). Consiste basicamente de uma 
caixa "reservatório", montada na parte superior da estrutura, com autonomia para preenchimento de, aproximadamente, $100 \mathrm{~m}$ de drenos (Figura 1).

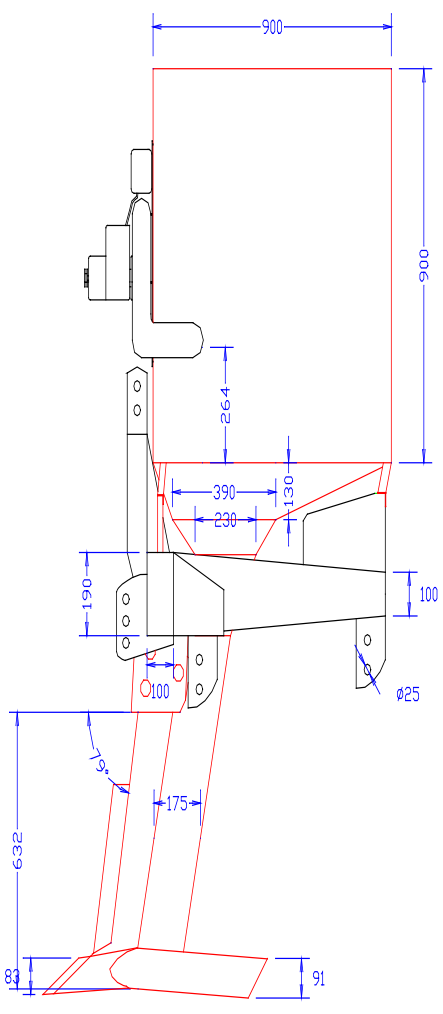

Figura 1. Vista lateral do protótipo (dimensões em mm).

Como material poroso, preferiu-se utilizar a casca de arroz. Este material possuir 10,4\% de sílica em sua constituição. Isso torna difícil a decomposição, o que torna seu descarte um problema em regiões produtoras de arroz.

Nesta área analisou-se a composição textural, teor de matéria orgânica, umidade e densidade do solo e a resistência do solo a penetração, em cada uma das profundidades trabalhadas. O solo da região é classificado como Podzólico Vermelho-Amarelo.

$\mathrm{O}$ experimento foi montado seguindo um delineamento experimental do tipo fatorial de $2 \mathrm{x}$ 4 , para velocidade e profundidade, respectivamente. Foram realizadas 3 repetições. Testou-se o esforço de tração para determinar a velocidade ideal de trabalho para o protótipo. Os seguintes aspectos foram avaliados: força de tração, potência requerida, velocidade de deslocamento, consumo de combustível e área mobilizada no solo, para as profundidades de $0,15 \mathrm{~m}, 0,30 \mathrm{~m}, 0,45 \mathrm{~m}$ e $0,60 \mathrm{~m}$.

Na medição da força de tração utilizaram-se dois tratores, sendo um responsável pela tração (MF
95X) e o outro como porta-ferramenta (MF 265, 4x2 TDA). O trator porta-ferramenta permanecia ligado em neutro, sendo responsável apenas pelo controle de profundidade. Para a leitura dos esforços, instalouse uma célula de carga presa entre os tratores por correntes fixadas nas barras de tração. Foi utilizada uma célula de marca KRATOS, modelo KLS, com capacidade para $50 \mathrm{kN}$, leitura digital e constante de leitura igual a 0,5 (a leitura da célula eqüivale ao dobro do esforço real), sendo esta previamente aferida. Os valores foram lidos durante um percurso de $50 \mathrm{~m}$, com três repetições para cada uma das quatro profundidades de trabalho. Coletaramse também, nas mesmas condições, os dados de esforço de tração para o trator sem implemento, para que fossem descontados nos cálculos de esforço do implemento (esforço relativo ao implemento $=$ esforço do trator mais implemento trabalhando esforço do trator mais implemento levantado).

Utilizou-se duas velocidades de deslocamento nos testes de esforço requerido, relativas às marchas $2^{\mathrm{a}}$ reduzida $\left(\mathrm{V} 1=2,7 \mathrm{~km} \mathrm{~h}^{-1}\right)$ e $3^{\mathrm{a}}$ reduzida $(\mathrm{V} 2=4,9$ $\mathrm{km} \mathrm{h}^{-1}$ ), com o motor na rotação parcial de 1.700 rpm. A velocidade real foi determinada medindose, com um cronômetro digital, o tempo gasto para percorrer $50 \mathrm{~m}$.

O consumo de combustível foi determinado utilizando-se um sistema medidor, da marca ISTEC, com leituras em galões e precisão de 0,001 galão, instalado no circuito de alimentação do motor. Este sistema consistia em dois medidores analógicos. Um deles foi instalado entre o filtro e a bomba injetora, sendo responsável pela medida total do combustível enviado à bomba. Já o outro era encarregado de medir o retorno de combustível da bomba e dos bicos de injeção do motor.

A profundidade de trabalho do implemento e a força de tração média foram medidas. Por meio da Equação 1, determinou-se o acréscimo de força requerida para cada centímetro de penetração da haste.

$\mathrm{Fm}=\frac{\mathrm{Fm}}{\mathrm{H}}$

em que

Fm = acréscimo de força $\left(\mathrm{N} \mathrm{m}^{-1}\right)$;

$\mathrm{Fm}=$ força de tração média $(\mathrm{N})$; e

$\mathrm{H}=$ profundidade de trabalho $(\mathrm{m})$. 
A potência disponível na barra de tração foi determinada utilizando-se a velocidade média de deslocamento do trator e a força média, conforme a Equação 2:

$$
\mathrm{Pb}=\frac{F m V m}{3.600}
$$

em que

$\mathrm{Pb}=$ potência disponível na barra de tração $(\mathrm{kW})$;

$\mathrm{Fm}=$ força de tração média $(\mathrm{N})$; e,

$\mathrm{Vm}=$ velocidade média de deslocamento $\left(\mathrm{km} \mathrm{h}^{-1}\right)$.

O consumo específico de combustível foi calculado pela Equação 3:

$$
\mathrm{Ce}=D \frac{C h}{P b}
$$

em que

$\mathrm{Ce}=$ consumo específico de combustível $\left(\mathrm{gkW}^{-1} \mathrm{~h}^{-1}\right)$;

$\mathrm{D}=$ massa específica do combustível $\left(\mathrm{gL}^{-1}\right)$;

$\mathrm{Ch}=$ consumo horário de combustível $\left(\mathrm{Lh}^{-1}\right) ; \mathrm{e}$

$\mathrm{Pb}=$ potência na barra de tração $(\mathrm{kW})$.

\section{RESULTADOS E DISCUSSÃO}

As análises mostraram que o solo possui textura muito argilosa em todas as profundidades trabalhadas, com distribuição uniforme da quantidade de argila no perfil, como pode ser observado no Quadro 1. Os dados de índice de cone apresentaram diferenças significativas, variando de $0,123 \mathrm{MPa}$ à $0,384 \mathrm{MPa}$ para as profundidades de $0,15 \mathrm{~m}$ e $0,60 \mathrm{~m}$, respectivamente. Essa variação pode ocorrer devido à diferença de teor de água do solo, fator que possui grande influência sobre este parâmetro. Já a densidade e textura do solo não apresentaram uma diferenciação que justificasse o aumento do índice de cone com o acréscimo da profundidade (Quadro 1). No momento da realização dos testes, os teores de água no solo foram $25,26 \%, 28,96 \%, 30,98 \%$ e $32,11 \%$, para as profundidades de $0,15 \mathrm{~m}, 0,30 \mathrm{~m}, 0,45 \mathrm{~m}$ e $0,60 \mathrm{~m}$, respectivamente.

As velocidades de deslocamento observadas em campo e consumo de combustível, para as profundidades trabalhadas são apresentadas na Quadro 2. Para a velocidade 1 (2 $2^{\mathrm{a}}$ reduzida) a velocidade média foi $2,7 \mathrm{~km} \mathrm{~h}^{-1}$, para a velocidade 2 (3a reduzida), foi em média $4,9 \mathrm{~km} \mathrm{~h}^{-1}$.

A análise dos valores de força de tração média em função da profundidade de trabalho e da velocidade de deslocamento do conjunto tratorimplemento e implemento são apresentadas nos Quadros 3 e 4 . Pode-se observar, em ambos

Quadro 1. Características do solo nas profundidades

\begin{tabular}{lcccc}
\hline & \multicolumn{4}{c}{ Profundidades (m) } \\
\cline { 2 - 5 } Propriedades & 0,15 & 0,30 & 0,45 & 0,60 \\
\hline Argila (\%) & 76 & 75 & 76 & 77 \\
Silte (\%) & 21 & 22 & 20 & 21 \\
Areia grossa (\%) & 2 & 2 & 1 & 2 \\
Areia fina (\%) & 1 & 1 & 3 & 2 \\
Matéria Orgânica (\%) & 3,02 & 2,88 & 1,54 & 1,13 \\
Índice de cone (MPa) & $0,123 \mathrm{a}^{*}$ & $0,219 \mathrm{~b}$ & $0,319 \mathrm{c}$ & $0,384 \mathrm{~d}$ \\
Densidade do solo $\left(\mathrm{g} \mathrm{cm}^{-3}\right)$ & 1,13 & 1,13 & 1,15 & 1,16 \\
* Médias seguidas de pelo menos uma mesma letra minúscula, na linha, não diferem estatisticamente entre si, pelo \\
teste de Tukey, a 5\% de probabilidade.
\end{tabular}

\begin{tabular}{|c|c|c|c|c|c|}
\hline \multirow{2}{*}{ Marchas } & \multicolumn{4}{|c|}{ Profundidades (m) } & \multirow{2}{*}{$\begin{array}{l}\text { Médias } \\
\left(\mathrm{km} \mathrm{h}^{-1}\right)\end{array}$} \\
\hline & 0,15 & 0,30 & 0,45 & 0,60 & \\
\hline $2^{\mathrm{a}}$ reduzida & 2,92 & 2,83 & 2,73 & 2,59 & 2,70 \\
\hline $3^{\mathrm{a}}$ reduzida & 5,24 & 5,05 & 4,78 & 4,67 & 4,90 \\
\hline
\end{tabular}

Quadro 2. Velocidades médias $\left(\mathrm{km} \mathrm{h}^{-1}\right)$ observadas em campo para as profundidades trabalhadas 
os Quadros, que a variação das profundidades de trabalho adotadas induziu, na maioria dos tratamentos, a diferenças significativas para a força de tração média. Isso não foi observado para as profundidades $0,45 \mathrm{~m}$ e $0,60 \mathrm{~m}$, para a velocidade de $4,9 \mathrm{~km} \mathrm{~h}^{-1}$. Nota-se ainda, que a variação na força de tração requerida foi significativa nas profundidades de $0,15 \mathrm{~m}$ e $0,45 \mathrm{~m}$, quando se passou de $2,7 \mathrm{~km} \mathrm{~h}^{-1}$ para $4,9 \mathrm{~km} \mathrm{~h}^{-1}$.

Os valores de força de tração média requeridos pelo implemento, tomando como exemplo a profundidade de $0,45 \mathrm{~m}$, para a velocidade 2,7 $\mathrm{km} \mathrm{h}^{-1}$, foi de 10313,36 N (Quadro 4). Esse valor é menor do que $18467,7 \mathrm{~N}$, encontrado por Garcia (1993), para a profundidade de $0,40 \mathrm{~m}$, subsolador com haste parabólica e velocidade de 2,34 $\mathrm{km} \mathrm{h}^{-1}$. Também Lanças (1988) encontrou um valor superior, 18829,3 N, para hastes retas inclinadas. Isso pode ser atribuído ao fato de que, normalmente, esses valores são obtidos a teores de água do solo mais baixos do que os utilizados nos trabalhos com subsoladores com torpedo.
Observou-se que a relação (força requerida)/ (profundidade de trabalho) não apresentou diferenças significativas com o acréscimo da profundidade de trabalho para a velocidade de 2,7 $\mathrm{km} \mathrm{h}^{-1}$ (Quadro 5). Porém, para a velocidade de $4,9 \mathrm{~km} \mathrm{~h}^{-1}$, essa relação apresentou diferença significativa para a profundidade de $0,15 \mathrm{~m}$. Isso ocorreu, provavelmente, porque em uma das leituras de força, para a velocidade V2 na profundidade de $0,15 \mathrm{~m}$, o valor observado foi $68 \%$ superior aos demais, acabando por levar a uma diferença estatisticamente significativa também quando se comparou as duas velocidades. A média geral da relação força/profundidade foi de $25830 \mathrm{~N} \mathrm{~m}^{-1}$, mantendo-se dentro do intervalo estipulado pela ASAE (1990) para solos argilosos que é de $17500 \mathrm{~N} \mathrm{~m}^{-1}$ a $28000 \mathrm{~N} \mathrm{~m}^{-1}$. O valor encontrado de $25830 \mathrm{~N} \mathrm{~m}^{-1}$ situa-se mais próximo do limite superior do intervalo, porque o solo da área onde se realizou os testes é muito argiloso.

As potências requeridas pela resistência ao rolamento das rodas do trator foram de $1,7 \mathrm{~kW}$ para a velocidade de $2,7 \mathrm{~km} \mathrm{~h}^{-1}(\mathrm{~V} 1)$ e de $3,1 \mathrm{~kW}$ para $4,9 \mathrm{~km} \mathrm{~h}^{-1}$ (V2).

Quadro 3. Força de tração média requerida pelo conjunto trator implemento $(\mathrm{N})$ em relação às profundidades $(\mathrm{m})$ e velocidades de trabalho $\left(\mathrm{km} \mathrm{h}^{-1}\right)$

\begin{tabular}{ccc}
\hline Profundidades & \multicolumn{3}{c}{ Velocidades } \\
\cline { 2 - 3 } & 2,70 & 4,90 \\
\hline 0,15 & $5583,45 \mathrm{Aa}^{*}$ & $7466,40 \mathrm{Ab}$ \\
0,30 & $9663,16 \mathrm{Ba}$ & $9970,45 \mathrm{Ba}$ \\
0,45 & $12363,36 \mathrm{Ca}$ & $15200,85 \mathrm{Cb}$ \\
0,60 & $15194,31 \mathrm{Da}$ & $15897,15 \mathrm{Ca}$ \\
\hline Médias (N) & 10701,07 & 12133,71 \\
\hline CV $(\%)$ & 8,64 &
\end{tabular}

* Médias seguidas de pelo menos uma mesma letra minúscula, em linha, e de letra maiúscula, na coluna, não diferem estatisticamente entre si, pelo teste de Tukey, a 5\% de probabilidade.

Quadro 4. Força de tração média $(\mathrm{N})$ requerida pelo implemento em relação à profundidade $(\mathrm{m})$ e velocidade de trabalho $\left(\mathrm{km} \mathrm{h}^{-1}\right)$

\begin{tabular}{ccc}
\hline \multirow{2}{*}{ Profundidades } & \multicolumn{2}{c}{ Velocidades } \\
\cline { 2 - 3 } & 2,70 & 4,90 \\
\hline 15 & $3533,45 \mathrm{Aa}^{*}$ & $5280,40 \mathrm{Ab}$ \\
30 & $7613,16 \mathrm{Ba}$ & $7784,45 \mathrm{Ba}$ \\
45 & $10313,36 \mathrm{Ca}$ & $13014,85 \mathrm{Cb}$ \\
60 & $13144,31 \mathrm{Da}$ & $13711,15 \mathrm{Ca}$ \\
\hline Médias (N) & 8651,07 & 9947,71 \\
\hline CV $(\%)$ & 10,60 & \\
\hline
\end{tabular}

* Médias seguidas de pelo menos uma mesma letra minúscula, em linha, e de letra maiúscula, na coluna, não diferem estatisticamente entre si, pelo teste de Tukey, a $5 \%$ de probabilidade. 
Nota-se, pela análise do Quadro 6, que para todas as profundidades houve influência significativa da velocidade. Quando é analisado o efeito da profundidade sobre a potência, para a velocidade média de $2,7 \mathrm{~km} \mathrm{~h}^{-1}$, vê-se que o efeito da profundidade não é verificado entre as profundidades de $0,15 \mathrm{~m}$ e $0,30 \mathrm{~m}, 0,30 \mathrm{~m}$ e 0,45 $\mathrm{m}$ e $0,45 \mathrm{~m}$ com $0,60 \mathrm{~m}$. Na velocidade de $4,9 \mathrm{~km}$ $\mathrm{h}^{-1}$, apenas nas profundidades de 0,45 e $0,60 \mathrm{~m}$ não houve diferença significativa, pelo fato dos teores de água do solo estarem muito próximos no momento dos testes $(30,98 \%$ e $32,11 \%$, respectivamente).
As potências requeridas pelo implemento, mostradas no Quadro 7, são semelhantes às encontradas por Garcia (1993), quando comparadas para uma mesma profundidade.

Os resultados de consumo específico de combustível, expostos no Quadro 8, tiveram um decréscimo com o aumento da profundidade. Entretanto, não diferenciando significativamente entre as profundidades de $0,45 \mathrm{~m}$ e $0,60 \mathrm{~m}$, para ambas as velocidades de trabalho. A profundidade de $0,15 \mathrm{~m}$ foi a que apresentou maior valor, devido à baixa potência exigida da máquina.

Quadro 5. Relação força/profundidade $\left(\mathrm{N} \mathrm{m}^{-1}\right)$ em relação às profundidades $(\mathrm{m})$ e velocidades de trabalho $\left(\mathrm{km} \mathrm{h}^{-1}\right)$

\begin{tabular}{ccc}
\hline Profundidades & \multicolumn{2}{c}{ Velocidades } \\
\cline { 2 - 3 } & 2,70 & 4,90 \\
\hline 0,15 & $2355 \mathrm{Aa}^{*}$ & $3520 \mathrm{Ab}$ \\
0,45 & $2291 \mathrm{Aa}$ & $2892 \mathrm{ABa}$ \\
0,30 & $2537 \mathrm{Aa}$ & $2594 \mathrm{Ba}$ \\
0,60 & $2190 \mathrm{Aa}$ & $2285 \mathrm{Ba}$ \\
\hline Médias $\left(\mathrm{N} \mathrm{m}^{-1}\right)$ & 2344 & 2823 \\
\hline $\mathrm{CV}(\%)$ & 13,69 & \\
\hline
\end{tabular}

* Médias seguidas de pelo menos uma mesma letra minúscula, em linha, e de letra maiúscula, na coluna, não diferem estatisticamente entre si, pelo teste de Tukey, a 5\% de probabilidade.

Quadro 6. Análise estatística das Potências médias $(\mathrm{kW})$ requeridas pelo conjunto trator implemento em função das velocidades $\left(\mathrm{km} \mathrm{h}^{-1}\right)$ e profundidades de trabalho $(\mathrm{m})$

\begin{tabular}{ccc}
\hline Profundidades & \multicolumn{2}{c}{ Velocidades } \\
\cline { 2 - 3 } & 2,70 & 4,90 \\
\hline 0,15 & $4,53 \mathrm{Aa}^{*}$ & $10,85 \mathrm{Ab}$ \\
0,30 & $7,59 \mathrm{ABa}$ & $13,98 \mathrm{Bb}$ \\
0,45 & $9,38 \mathrm{BCa}$ & $20,18 \mathrm{Cb}$ \\
0,60 & $10,92 \mathrm{Ca}$ & $20,69 \mathrm{Cb}$ \\
\hline Médias $(\mathrm{kW})$ & 8,11 & 16,42 \\
\hline CV $(\%)$ & 10,69 & \\
\hline
\end{tabular}

* Médias seguidas de pelo menos uma mesma letra minúscula, em linha, e de letra maiúscula, na coluna, não diferem estatisticamente entre si, pelo teste de Tukey, a 5\% de probabilidade.

Quadro 7. Análise estatística das potências médias $(\mathrm{kW})$, requeridas pelo implemento, em relação às velocidades $\left(\mathrm{km} \mathrm{h}^{-1}\right)$ e profundidades de trabalho $(\mathrm{m})$

\begin{tabular}{ccc}
\hline Profundidades & \multicolumn{2}{c}{ Velocidades } \\
\cline { 2 - 3 } & 2,70 & 4,90 \\
\hline 0,15 & $2,86 \mathrm{Aa}^{*}$ & $7,67 \mathrm{Ab}$ \\
0,30 & $5,98 \mathrm{Ba}$ & $10,91 \mathrm{Bb}$ \\
0,45 & $7,83 \mathrm{BCa}$ & $17,27 \mathrm{Cb}$ \\
0,60 & $9,45 \mathrm{Ca}$ & $17,85 \mathrm{Cb}$ \\
\hline Médias $(\mathrm{kW})$ & 6,53 & 13,43 \\
\hline CV $(\%)$ & 12,64 & \\
\hline
\end{tabular}

* Médias seguidas de pelo menos uma mesma letra minúscula, em linha, e de letra maiúscula, na coluna, não diferem estatisticamente entre si, pelo teste de Tukey, a 5\% de probabilidade. 
Quadro 8. Resultados da análise estatística, do Consumo específico de combustível (g.kW-1 $\mathrm{h}^{-1}$ ) em relação às profundidades $(\mathrm{m})$ e velocidades de trabalho $\left(\mathrm{km} \mathrm{h}^{-1}\right)$

\begin{tabular}{cccc}
\hline Profundidades & \multicolumn{2}{c}{ Velocidades } & Médias \\
\cline { 2 - 3 } & 2,70 & 4,90 & 1029,9 \\
\hline 0,15 & $1213,5 \mathrm{Aa}^{*}$ & $846,2 \mathrm{Ab}$ & 688,6 \\
0,30 & $766,8 \mathrm{Ba}$ & $610,4 \mathrm{Ba}$ & 474,6 \\
0,45 & $574,1 \mathrm{BCa}$ & $375,0 \mathrm{Cb}$ & 394,9 \\
0,60 & $415,7 \mathrm{Ca}$ & $374,2 \mathrm{Ca}$ & 647,00 \\
\hline Médias $\left({\left.\mathrm{g} . \mathrm{kW}^{-1} \mathrm{~h}^{-1}\right)}_{\mathrm{CV}(\%)}^{742,53}\right.$ & 551,4741 & \\
\hline
\end{tabular}

* Médias seguidas de pelo menos uma mesma letra minúscula, em linha, e de letra maiúscula, na coluna, não diferem estatisticamente entre si, pelo teste de Tukey, a 5\% de probabilidade.

Lanças (1988), utilizando um subsolador com haste reta inclinada, trabalhando a 0,40 $\mathrm{m}$ de profundidade, encontrou um valor de consumo específico de $0,74 \mathrm{~L} \mathrm{~kW}^{-1} \mathrm{~h}^{-1}$. Este valor é próximo dos valores obtidos na profundidade de $0,45 \mathrm{~m}\left(0,68 \mathrm{~L} \mathrm{~kW}^{-1} \mathrm{~h}^{-1}\right)$ para a velocidade de $2,7 \mathrm{~km} \mathrm{~h}^{-1}$ e a $0,30 \mathrm{~m}^{-1}\left(0,73 \mathrm{~L} \mathrm{~kW}^{-1}\right.$ $\mathrm{h}^{-1}$ ) para a velocidade de $4,9 \mathrm{~km} \mathrm{~h}^{-1}$. A densidade do combustível utilizada para o cálculo foi de $837,91 \mathrm{~g} \mathrm{~L}^{-1}$, para a temperatura de $35{ }^{\circ} \mathrm{C}$ observada no campo.

\section{CONCLUSÕES}

- A maior força requerida pelo implemento $(13711,1 \mathrm{~N})$, bem como a maior potência $(17,85 \mathrm{~kW})$, foi observada para a velocidade de $4,9 \mathrm{~km} \mathrm{~h}^{-1}$, na profundidade de $60 \mathrm{~cm}$, sendo menores do que as encontradas na literatura para subsoladores com hastes retas inclinadas;

- A força média requerida pelo protótipo foi de 9299,4 N, sendo menor do que a encontrada na literatura;

- A média da potência requerida pelo implemento, $9,98 \mathrm{~kW}$, e pelo conjunto trator implemento, $12,26 \mathrm{~kW}$, está próxima aos valores encontrados na literatura; e

- Em média, a relação força de tração/ profundidade de trabalho encontra-se dentro do limite estabelecido pela ASAE para solos argilosos.

\section{REFERÊNCIAS BIBLIOGRÁFICAS}

ASAE. Standards 1990. 37_a ed., p.287, 1990. $717 \mathrm{p}$.

BRIDGES, R.C., SMITH, E.M. Amethod for determining the total energy imput for agricultural practices. Transactions of the ASAE, St. Joseph, v.22, n.4, p.781-784, 1979.

GARCIA, A.S. Subsolador: desempenho energético e operacional em função da velocidade de deslocamento e do teor de água no solo. Botucatu: UNESP, 1993. 141f. Dissertação (mestrado em agronomia) - Universidade Estadual Paulista, 1993.

GROTTA, D.C.C., CARLOS, A.L., KLINGER, E. A.F., GUSTAVO, B.B., REIS, N. dos, DA SILVA, R. P. Subsolador: avaliação do desempenho em função da velocidade de trabalho e espaçamento entre hastes. Acta Scientiarum. Agronomy. Maringá, v.26, n.1, p.21-26, 2004.

HUNT, D. Maquinaria Agrícola: rendimento económicos, costos, operaciones, potencia e selección de equipo. 7 ed. México: Limusa, 1986. 451p.

LANÇAS, K.P., Subsolador: desempenho em função das formas geométricas das hastes, tipos de ponteiras e número de hastes. Botucatu, UNESP, 1988. 171f. Tese (doutorado em Agronomia) - Universidade Estadual Paulista, 1988. 
MARTUCCI, F.G. Escarificador no preparo do solo. Granja, Porto Alegre, v.6, n.449, p.44-46, 1985.

ORLANDO, R.C. Desenvolvimento de um subsolador com torpedo e dispositivo para inserção de material poroso em drenos livres. Viçosa, MG: UFV, 1999. 66 f. Dissertação (Mestrado em Engenharia agrícola) - Universidade Federal de Viçosa, Viçosa.1999.

PLASSE, R., RAGHAVAN, G.S.V., McKYES, E. Simulation of narrow blade performance in different soils. Transation of the ASAE, St. Joseph, v.28, n.4, p.1007 - 1012, 1985.

SANTOS FILHO, A. G. Subsolador: Desempenho energético e operacional em função da velocidade de deslocamento e do teor de água no solo. Botucatu: UNESP, 1993.122 f. Dissertação (mestrado em agronomia) - Universidade Estadual Paulista, 1993.

SUMMERS, J.D., KHALILIAN, A., BATCHELDER, D.G. Draft relationships for primary tillage in Oklahoma soils. Transaction of the ASAE, St. Joseph, v.29, n 1, p.37-39, 1986.

SALVADOR, N.; BENEZ, S.H.; MION, R.L. Demanda energética em diferentes sistemas de preparo periódico do solo antes e depois da subsolagem. Revista Ciência Agronômica, Fortaleza, v.39, n.03, p.378-383, 2008. 